\title{
COVID-19: ¿UNA OPORTUNIDAD DE CAMBIO PARA EL PERIODISMO DEPORTIVO? ANÁLISIS DE LA COBERTURA DEPORTIVA DE EL PAÍS, EL MUNDO Y LA VANGUARDIA DURANTE EL ESTADO DE ALARMA
}

COVID-19: An opportunity for change in sports journalism? Analysis of the sports coverage of El País, El Mundo and La Vanguardia during the state of alarm

\author{
Victor HASBANI KERMANCHAHI ${ }^{1}$, Xavier RAMÓN¹ y Sergi CORTIÑAS-ROVIRA ${ }^{1}$ \\ 1 Universitat Pompeu Fabra (España)
}

\section{Resumen}

El periodismo deportivo se ha caracterizado por la uniformidad y la baja diversidad temática, que se traduce en la excesiva focalización en determinados deportes, competiciones y protagonistas con alta capacidad de atracción económica. La crisis del Covid-19 ha ofrecido al periodismo deportivo la oportunidad de reexaminar sus estrategias y articular una agenda distintiva. A través del análisis del contenido de 1.409 piezas y 195 portadas, se examina la cobertura desarrollada por El País, El Mundo y La Vanguardia durante los primeros 65 días del estado de alarma, con el objetivo de determinar si los periódicos aprovecharon la crisis para desarrollar un periodismo deportivo más diverso. La crisis del Covid-19 no modificó las prioridades existentes, sino que intensificó la atención sobre aquellos deportes que ya acumulaban más poder mediático. En un escenario sin competiciones, la atención sobre el fútbol masculino y profesional permaneció intacta. La 'futbolización' contrasta con un escaso tratamiento del impacto de la pandemia sobre otras disciplinas minoritarias y el deporte femenino y adaptado. La prensa también fue ajena a varias temáticas, personajes y perspectivas de importancia a nivel mundial. Las disparidades en la agenda conllevan repercusiones sociales, económicas y deportivas de primer orden.

Palabras clave: periodismo deportivo; Covid-19; diversidad de la agenda; prensa; estado de alarma.

\begin{abstract}
Sports journalism has been characterized by uniformity and low thematic diversity, which translates into excessive focus on certain sports, competitions and protagonists with a high capacity for economic attraction. The Covid-19 crisis has offered sports journalism the opportunity to reexamine its strategies and articulate a distinctive agenda. Through content analysis of 1,409 pieces and 195 front pages, the coverage developed by El País, El Mundo and La Vanguardia during the first 65 days of the state of alarm is examined, with the goal of determining whether newspapers took advantage of the crisis to develop a more diverse sports journalism. The Covid-19 crisis did not modify the existing priorities, but rather intensified attention on those sports that already accumulated more media power. In a landscape without competitions, the focus on men's and professional football remained intact. 'Footballization' contrasts with a scant treatment of the impact of the pandemic on other minority disciplines and women's and disability sport. The press was also oblivious to various themes protagonists and perspectives of worldwide importance. The disparities on the agenda carry major social, economic and sports repercussions.
\end{abstract}

Keywords: sports journalism; Covid-19; agenda diversity; press; state of alarm. 


\section{Introducción}

Tradicionalmente, el periodismo deportivo ha sido considerado como el 'hermano pequeño' de la profesión o el 'departamento de juguetes' (toy department) de las redacciones (Rowe 2007). Sin embargo, en el contexto presente el periodismo deportivo "desempeña, como manifestación de la cultura contemporánea, un papel estratégico en la actual estructura de los medios de comunicación" (Rojas-Torrijos 2018, 134). En los últimos años, el periodismo ha atravesado cambios estructurales de primer orden, entre los que cabe destacar el descenso de la inversión publicitaria, la creciente competencia por parte de múltiples actores y el colapso de los modelos de negocio tradicionales (Waisbord 2019). En este contexto de inestabilidad, los contenidos deportivos se han convertido en un activo fundamental para atraer y retener a la audiencia. Como señala Serazio $(2019$, 4), "en una era de fragmentación cultural, polarización política y la distracción que deriva de la abundancia de medios, el deporte representa una de las últimas instituciones que unifican a las masas".

Más allá de su innegable valor económico para los medios, la información deportiva juega un papel clave en la transmisión de valores y en la formación de identidades individuales y colectivas. El fuerte impacto cultural y social de los contenidos deportivos exige, por parte de los medios de comunicación, una aproximación completa, diversa y responsable a esta área de especialización. Desde la óptica de la teoría normativa de los medios, la diversidad implica informar a los ciudadanos sobre un amplio espectro de temáticas y visibilizar a los diversos grupos sociales (Steiner, Magin y Stark 2019). Entre las obligaciones éticas de los medios respecto a la información deportiva se encuentra la necesidad de fomentar la diversidad en la agenda informativa (Ramon-Vegas et al. 2020).

Pese a esta claridad normativa, el periodismo deportivo se ha caracterizado por una falta de diversidad, que se traduce en la excesiva focalización en determinados deportes, competiciones y protagonistas con alta capacidad de atracción económica. El seguimiento rutinario de la actualidad deportiva en Europa pivota alrededor de las grandes ligas de fútbol y los torneos continentales como la UEFA Champions League, eventos capaces de generar una atención mediática constante en todo tipo de medios y espacios informativos, incluso en períodos de menor intensidad competitiva. La dependencia respecto a los eventos deportivos es un componente esencial de esta área de especialización. ¿Pero qué sucede cuando de forma inesperada el periodismo deportivo pierde a su principal ingrediente?

La expansión del Covid-19 ha tenido un impacto sin precedentes en el mundo del deporte. Con el objetivo de garantizar el cumplimiento de las medidas de distanciamiento social y salvaguardar la salud de los deportistas frente a la expansión del virus, se ha producido una cancelación o suspensión masiva de las competiciones deportivas en todo el mundo (Horky 2021; Parnell et al. 2020; Tovar 2021). Más allá de torneos nacionales, el Covid-19 ha tenido un impacto de primer orden sobre los mega-eventos deportivos a nivel global. Los Juegos Olímpicos y Paralímpicos de Tokio 2020 y la Eurocopa de fútbol de 2020 - que por primera vez se debía celebrar en 12 ciudades europeas distintas - han sido pospuestos hasta 2021 (Tabla 1). En total, se han cancelado o suspendido más de 250 eventos en todos los continentes (Mena 2020). Este inesperado escenario ha ofrecido al periodismo deportivo la oportunidad de reexaminar sus estrategias y articular una agenda distintiva.

\begin{tabular}{c|c|c|c|c} 
Competición & Deporte & Estado actual & $\begin{array}{c}\text { Fecha de } \\
\text { suspensión / } \\
\text { cancelación }\end{array}$ & $\begin{array}{c}\text { Nueva fecha de } \\
\text { celebración }\end{array}$ \\
\hline $\begin{array}{c}\text { Campeonato } \\
\text { Europeo de }\end{array}$ & Atletismo & Cancelada & $23 / 03 / 2020$ & $\begin{array}{c}15-21 \text { de agosto } \\
\text { de } 2022 \text { (Múnich) }\end{array}$ \\
$\begin{array}{c}\text { Atletismo París } \\
2020\end{array}$ & & & \\
\hline
\end{tabular}




\begin{tabular}{|c|c|c|c|c|}
\hline $\begin{array}{c}\text { Campeonato } \\
\text { Europeo de Para } \\
\text { Atletismo } \\
\text { Bydgoszcz } 2020\end{array}$ & Atletismo & Pospuesta & $\begin{array}{c}\text { Debía iniciarse el } \\
02 / 06 / 2020\end{array}$ & Por determinar \\
\hline $\begin{array}{c}\text { National } \\
\text { Basketball } \\
\text { Association (NBA) }\end{array}$ & Baloncesto & Reanudada & $11 / 03 / 2020$ & $30 / 07 / 2020$ \\
\hline Euroliga & Baloncesto & Cancelada & $25 / 05 / 2020$ & Temporada 20-21 \\
\hline Liga Endesa & Baloncesto & $\begin{array}{l}\text { Reanudada y } \\
\text { finalizada }\end{array}$ & $16 / 03 / 2020$ & $\begin{array}{c}17 / 06 / 2020- \\
30 / 06 / 2020\end{array}$ \\
\hline Liga ASOBAL & Balonmano & Finalizada & $04 / 05 / 2020$ & Temporada 20-21 \\
\hline $\begin{array}{l}\text { Major League } \\
\text { Baseball (MLB) }\end{array}$ & Béisbol & Pospuesta & $\begin{array}{c}\text { Debía iniciarse el } \\
26 / 03 / 2020\end{array}$ & $23 / 07 / 2020$ \\
\hline Vuelta a España & Ciclismo & Pospuesta & $\begin{array}{c}\text { Debía iniciarse el } \\
14 / 08 / 2020 \\
\end{array}$ & $\begin{array}{c}20 / 10 / 2020- \\
08 / 11 / 2020 \\
\end{array}$ \\
\hline Tour de Francia & Ciclismo & Pospuesta & $\begin{array}{c}\text { Debía iniciarse el } \\
27 / 06 / 2020\end{array}$ & $\begin{array}{c}29 / 08 / 2020- \\
20 / 09 / 2020\end{array}$ \\
\hline La Liga & Fútbol & $\begin{array}{l}\text { Reanudada y } \\
\text { finalizada }\end{array}$ & $12 / 03 / 2020$ & $\begin{array}{c}08 / 06 / 2020- \\
19 / 07 / 2020\end{array}$ \\
\hline Liga Iberdrola & Fútbol & Finalizada & $06 / 05 / 2020$ & Temporada 20-21 \\
\hline $\begin{array}{l}\text { Bundesliga } \\
\text { (Alemania) }\end{array}$ & Fútbol & $\begin{array}{l}\text { Reanudada y } \\
\text { finalizada }\end{array}$ & $17 / 03 / 2020$ & $\begin{array}{c}16 / 05 / 2020- \\
27 / 06 / 2020\end{array}$ \\
\hline Ligue 1 (Francia) & Fútbol & Finalizada & $30 / 04 / 2020$ & Temporada 20-21 \\
\hline $\begin{array}{l}\text { Premier League } \\
\text { (Reino Unido) }\end{array}$ & Fútbol & $\begin{array}{l}\text { Reanudada y } \\
\text { finalizada }\end{array}$ & $13 / 03 / 2020$ & $\begin{array}{c}17 / 06 / 2020- \\
26 / 07 / 2020\end{array}$ \\
\hline Serie A (Italia) & Fútbol & $\begin{array}{l}\text { Reanudada y } \\
\text { finalizada }\end{array}$ & 09/03/2020 & $\begin{array}{c}13 / 06 / 2020- \\
02 / 08 / 2020 \\
\end{array}$ \\
\hline $\begin{array}{c}\text { UEFA } \\
\text { Champions } \\
\text { League }\end{array}$ & Fútbol & Reanudada & $13 / 03 / 2020$ & $07 / 08 / 2020$ \\
\hline Eurocopa 2020 & Fútbol & Pospuesta & $\begin{array}{c}\text { Debía iniciarse el } \\
12 / 06 / 2020\end{array}$ & $\begin{array}{c}11 / 06 / 2021- \\
11 / 07 / 2021\end{array}$ \\
\hline $\begin{array}{l}\text { Copa América de } \\
\text { Fútbol }\end{array}$ & Fútbol & Pospuesta & $\begin{array}{c}\text { Debía iniciarse el } \\
12 / 06 / 2020\end{array}$ & $\begin{array}{c}11 / 06 / 2021- \\
11 / 07 / 2021\end{array}$ \\
\hline $\begin{array}{c}\text { Masters de } \\
\text { Augusta }\end{array}$ & Golf & Pospuesta & $\begin{array}{c}\text { Debía iniciarse el } \\
09 / 04 / 2020\end{array}$ & $\begin{array}{c}12 / 11 / 2020- \\
15 / 11 / 2020\end{array}$ \\
\hline British Open & Golf & Cancelada & $\begin{array}{c}\text { Debía iniciarse el } \\
16 / 07 / 2020\end{array}$ & $\begin{array}{c}15 / 07 / 2021- \\
18 / 07 / 2021\end{array}$ \\
\hline $\begin{array}{c}\text { PGA } \\
\text { Championship }\end{array}$ & Golf & Pospuesta & $\begin{array}{c}\text { Debía iniciarse el } \\
14 / 05 / 2020\end{array}$ & $\begin{array}{c}06 / 08 / 2020- \\
09 / 08 / 2020 \\
\end{array}$ \\
\hline US Open & Golf & Pospuesta & $\begin{array}{c}\text { Debía iniciarse el } \\
18 / 06 / 2020\end{array}$ & $\begin{array}{c}17 / 09 / 2020- \\
20 / 09 / 2020\end{array}$ \\
\hline $\begin{array}{l}\text { National Hockey } \\
\text { League (NHL) }\end{array}$ & Hockey & Pospuesta & $12 / 03 / 2020$ & $01 / 08 / 2020$ \\
\hline MotoGP & Motor & Reanudada & $01 / 03 / 2020$ & $19 / 07 / 2020$ \\
\hline Formula 1 & Motor & Reanudada & $13 / 03 / 2020$ & $05 / 07 / 2020$ \\
\hline Juegos Olímpicos & Polideportivo & Pospuesta & $\begin{array}{c}\text { Debía iniciarse el } \\
24 / 07 / 2020\end{array}$ & $\begin{array}{c}23 / 07 / 2021- \\
08 / 08 / 2021\end{array}$ \\
\hline $\begin{array}{c}\text { Juegos } \\
\text { Paralímpicos }\end{array}$ & Polideportivo & Pospuesta & $\begin{array}{c}\text { Debía iniciarse el } \\
25 / 08 / 2020\end{array}$ & $\begin{array}{c}24 / 08 / 2021- \\
05 / 09 / 2021\end{array}$ \\
\hline $\begin{array}{c}\text { Torneo de las Seis } \\
\text { Naciones }\end{array}$ & Rugby & $\begin{array}{l}\text { Reanudada y } \\
\text { finalizada }\end{array}$ & 08/03/2020 & $\begin{array}{c}24 / 10 / 2020- \\
31 / 10 / 2020\end{array}$ \\
\hline $\begin{array}{c}\text { The Rugby } \\
\text { Championship }\end{array}$ & Rugby & Pospuesta & $\begin{array}{c}\text { Debía iniciarse el } \\
08 / 08 / 2020\end{array}$ & $\begin{array}{c}31 / 10 / 2020- \\
05 / 12 / 2020\end{array}$ \\
\hline Wimbledon & Tenis & Cancelada & $\begin{array}{c}\text { Debía iniciarse el } \\
29 / 06 / 2020 \\
\end{array}$ & $\begin{array}{c}28 / 06 / 2021- \\
11 / 07 / 2021 \\
\end{array}$ \\
\hline Roland Garros & Tenis & Pospuesta & $\begin{array}{c}\text { Debía iniciarse el } \\
24 / 05 / 2020\end{array}$ & $\begin{array}{c}20 / 09 / 2020- \\
04 / 10 / 2020\end{array}$ \\
\hline $\begin{array}{c}\text { Liga de } \\
\text { Waterpolo } \\
\text { masculina y } \\
\text { femenina }\end{array}$ & Waterpolo & Finalizada & $13 / 03 / 2020$ & Temporada 20-21 \\
\hline
\end{tabular}

Tabla 1. Afectación del Covid-19 sobre las principales competiciones deportivas. Fuente: Elaboración propia a partir de los sitios web de las competiciones. 
Durante la crisis sanitaria del Covid-19, la atención académica en el ámbito comunicativo se ha centrado en examinar cuestiones clave como la expansión de la desinformación a través de las redes sociales (Pulido et al. 2020; Salaverría et al. 2020), el papel de la radio como medio informativo (Rodero 2020) y el aumento de la producción y el consumo de noticias por parte de la ciudadanía (Casero-Ripollés 2020; Lázaro-Rodríguez y Herrera-Viedma 2020; Masip et al. 2020). Por su parte, Carvajal (2020) ha señalado las graves consecuencias del Covid-19 en el sector periodístico, entre las que cabe destacar el desplome de la inversión publicitaria, el descenso en la venta de ejemplares de prensa en papel, la aplicación de los ERTE, la precarización de la actividad profesional y las dificultades asociadas a la movilidad de los periodistas. Estos condicionantes han afectado no solamente a la cantidad sino también a la calidad de las informaciones publicadas.

Tomando en cuenta la importancia clave del deporte en las sociedades contemporáneas y en la actual estructura de los medios de comunicación, resulta especialmente relevante analizar de qué forma los principales medios generalistas han cubierto la necesidad informativa de los ciudadanos respecto al deporte. La investigación ahonda en varios debates: ¿Qué tipo de periodismo deportivo han desarrollado los principales medios generalistas en España? ¿Ha representado la pandemia una oportunidad para diversificar la apuesta informativa o bien ha reforzado las desigualdades existentes en la agenda deportiva? Para responder a estas cuestiones, se examina la cobertura desarrollada por El País, El Mundo y La Vanguardia durante los 65 días desde la aprobación del Real Decreto 463/2020, de 14 de marzo, por el que se declaró el estado de alarma para hacer frente al Covid-19 (España 2020).

La agenda en el periodismo deportivo: uniformidad y baja diversidad temática

Desde la perspectiva de la teoría de los campos, el periodismo deportivo es una especialización que tradicionalmente se ha inclinado hacia el capital económico en lugar de hacía el capital periodístico o cultural (Bourdieu 1998; English 2016). En la búsqueda constante del beneficio económico, el periodismo deportivo se ha configurado como un área caracterizada por la promoción de los deportistas, clubes y competiciones más importantes (Broussard 2020; English 2017; Rowe 2007). La fascinación y falta de distancia crítica respecto a la industria del deporte y a sus actores más relevantes ha llevado a los académicos a referirse al periodismo deportivo como "la mejor agencia de publicidad del mundo" (Schultz-Jørgensen 2005, 4).

Como señalan McCombs y Shaw (1972, 76), al escoger y difundir las noticias, los editores y redactores hacen que las audiencias "no sólo aprendan sobre una determinada cuestión, sino también sobre cuánta importancia deben otorgarle a este asunto a partir de la cantidad de información publicada y su posición". En el campo de la información deportiva, la selección, el énfasis y la exclusión de ciertos enfoques sobre la actualidad influye decisivamente sobre el conocimiento que la audiencia adquiere sobre el deporte y sus contextos relevantes. De forma habitual, la agenda deportiva se caracteriza por la uniformidad temática y la sobreabundancia de información sobre fútbol masculino y profesional, hecho que restringe de forma sustancial la diversidad de la información ofrecida a las audiencias (English 2014; Ramon-Vegas y Tulloch 2021; Rojas-Torrijos 2012; Sainz-de-Baranda, Barbero-González y Fernández-Fernández 2019).

Sin lugar a dudas, el fútbol es el deporte que aglutina más atención y cobertura a nivel global. No obstante, la 'tiranía del fútbol' o excesiva 'futbolización' de la agenda, que redunda en la repetición y uniformidad de las apuestas informativas, repercute en la infrarrepresentación de muchos otros deportes, disciplinas y protagonistas, incluido el deporte femenino (Franks y O'Neill 2016). A excepción de en algunos medios locales y regionales (Rojas-Torrijos y Jimeno 2019), resulta especialmente relevante remarcar que esta asimetría estructural de género en la información deportiva se produce tanto en medios deportivos (Herrero-Gutiérrez 2018; Sainz-de-Baranda 2014) como en los espacios dedicados al deporte en los medios generalistas (Calvo-Ortega y Gutiérrez San Miguel 2016; O’Neill y Mulready 2015). La invisibilidad del deporte adaptado en la agenda resulta 
aún más notoria, produciéndose incluso durante la celebración de los Juegos Paralímpicos (Solves et al. 2019).

Esta falta de diversidad, que limita las oportunidades de los ciudadanos de acceder a un amplio menú deportivo, se combina con otras carencias esenciales, que han caracterizado el periodismo deportivo como un ámbito "sin el rigor y la credibilidad de otras formas de periodismo" (Boyle 2017, 497). Entre estas prácticas cuestionables cabe destacar: el bufandismo y la constante disolución de las fronteras entre la información y la opinión; la prevalencia del rumor y la especulación; la frecuente espectacularización y trivialización de los contenidos; la polarización y orientación hacia la controversia y la confrontación; o la incapacidad de ahondar en los contextos políticos, económicos, sociales y tecnológicos de gran importancia que se relacionan con el deporte (Márquez-Ramírez y Rojas-Torrijos 2017; McEnnis 2020; Rowe 2017; Serazio 2019). La literatura académica también ha resaltado la limitada capacidad del periodismo deportivo convencional para ahondar en la historia del deporte desde múltiples ángulos, en contraste con otras publicaciones que apuestan por el periodismo deportivo lento y/o de largo formato (Ramon-Vegas y Tulloch 2016; 2021).

Covid-19: una oportunidad para diversificar la agenda deportiva

El estado de alarma derivado de la expansión del Covid-19 y la interrupción de las competiciones deportivas a nivel nacional e internacional ha obligado a los medios deportivos y generalistas a repensar sus estrategias informativas. Como recalca José Luis Rojas Torrijos, "este nuevo escenario ofrece la oportunidad a los periodistas deportivos de reinventarse y de probar nuevas formas de aproximarse a la realidad que circunda a los torneos y a sus protagonistas" (Rojas-Torrijos 2020). Como señala el mismo autor, el momento presente ofrece la posibilidad de diversificar la agenda: "dar al deporte más contexto social, cultural, económico y político en las informaciones", "buscar historias", "explorar nuevos ángulos" y, en definitiva, "mirar hacia un periodismo más ético para hacerlo más creíble y respetado" (Rojas-Torrijos 2020). En el contexto presente, es de gran relevancia comprender las particularidades de la apuesta informativa llevada a cabo por los medios generalistas con mayor audiencia en el contexto español, determinando si estas posibilidades para diversificar la agenda se han materializado o no.

\section{Metodología}

Esta investigación tiene como objetivo fundamental radiografiar el tratamiento deportivo que la prensa generalista de calidad española realizó durante la pandemia del Covid-19. La siguiente pregunta de investigación (PI) ha guiado el estudio:

PI: ¿De qué forma la prensa generalista española cubrió la información deportiva durante los primeros 65 días del estado de alarma por la crisis del Covid-19? ¿Aprovecharon los periódicos la oportunidad de desarrollar un periodismo deportivo más diverso?

Esta pregunta se concreta en dos objetivos específicos $(\mathrm{OE})$ :

OE1. Analizar la atención dedicada por El País, El Mundo y La Vanguardia a la actualidad deportiva durante los primeros 65 días del estado de alarma, tanto en portada como en las secciones de deporte.

OE2. Estudiar las cuestiones tratadas en la cobertura deportiva y determinar qué aspectos fueron olvidados o tratados de forma superficial.

Para dar respuesta a la pregunta de investigación y a los objetivos específicos, se ha empleado la técnica del análisis del contenido, que ha permitido leer, interpretar y hacer inferencias de forma sistemática del contenido periodístico que integra la muestra (Bryman 2016). El análisis del contenido ha sido la técnica de investigación prevalente en el ámbito de la comunicación deportiva. La combinación de las aproximaciones cuantitativa y cualitativa permite al investigador no circunscribirse únicamente a presentar números y porcentajes, superando la rigidez propia del análisis del contenido cuantitativo (Altheide 1996). Según su finalidad, el análisis del contenido aplicado no tiene un carácter puramente descriptivo, sino que es analítico, porque, aunque de forma 
sintética, se apuntan las causas, explicaciones e implicaciones de los fenómenos descritos (LópezAranguren 2016).

En esta investigación, la muestra ha sido escogida de forma no probabilística (Ruiz Olabuénaga, Aristegui y Melgosa 1998). Se han analizado tres medios de comunicación generalistas (El País, El Mundo y La Vanguardia) en sus versiones impresas, que se han seleccionado atendiendo a los criterios de trayectoria, audiencia y relevancia dentro del contexto mediático español. A diferencia de otros países europeos, España cuenta con prensa diaria deportiva, representada por cuatro periódicos de tirada nacional (Marca, AS, Mundo Deportivo y Sport) y varios medios de difusión regional. Sin embargo, resulta pertinente centrar la atención en la prensa generalista debido a su visibilidad e indudable peso en la configuración de la opinión pública en España. Conscientes de su gran poder para atraer a la audiencia, las cabeceras generalistas destinan un amplio espacio al deporte a través de secciones específicas, que destacan por la capacidad de transcender la 'futbolización' de los contenidos, por su mayor distancia crítica en comparación con la prensa especializada y por su habilidad de ahondar en el nexo entre el deporte y sus contextos relevantes (Márquez-Ramírez y Rojas-Torrijos 2017; Montero-Ramos 2017; Rojas-Torrijos 2012). Debido a su gran alcance y enfoque distintivo en el tratamiento deportivo, resulta fundamental examinar el comportamiento de la prensa de carácter general durante la crisis del Covid-19.

El País es la principal cabecera del grupo PRISA. Fundado en 1976, El País ha sido desde la transición democrática el diario generalista español de mayor influencia y difusión (Seoane y Sueiro 2004). Como señalan los datos del Marco General de Medios de la Asociación para la Investigación de los Medios de Comunicación, El País es el periódico con más audiencia en España (papel y visor digital/PDF), siendo únicamente superado por Marca (AIMC 2020, 46). Su impacto e influencia creciente en América Latina ha llevado al medio a autodenominarse 'El periódico global'.

El Mundo, fundado en 1989 y propiedad de Unidad Editorial, es el segundo periódico generalista con más audiencia en España (AIMC 2020). El periódico mantiene desde su creación una atención diaria preferente hacia el deporte, que se refleja en la sección diaria que dedica a esta área y en el suplemento deportivo del lunes, llamado "DXT". Finalmente, La Vanguardia, fundada en 1881 por Carlos y Bartolomé Godó, es el tercer periódico generalista con más audiencia, después de El País y El Mundo (AIMC 2020). Con sede en Barcelona, La Vanguardia ofrece a sus lectores una sección diaria sobre deportes, además del suplemento temático "Lunes Match".

En estos periódicos, se ha analizado la información deportiva publicada durante los primeros 65 días del estado de alarma en España (14 de marzo - 17 de mayo de 2020). Se ha escogido esta horquilla temporal debido a la magnitud de los desajustes que se produjeron durante este período en el panorama deportivo español y mundial (Horky 2021; Hutchins 2020; Parnell et al. 2020; Tovar 2021). A partir de entonces se ha empezado un paulatino progreso de vuelta a la normalidad que todavía se encuentra en curso. Para garantizar la exhaustividad en la recolección del material, se tuvieron en cuenta todas las piezas publicadas en las secciones de deporte de dichos medios durante el período temporal de la observación $(\mathrm{N}=1.409)$. El origen de las piezas es el siguiente: $E l$ Pais ( $\mathrm{n}=409)$, El Mundo ( $=206)$ y La Vanguardia $(\mathrm{n}=724)$. Dado que "en términos de audiencia e influencia social, la portada se conforma como el principal elemento de impacto de la prensa" (López-Rabadán y Casero-Ripollés 2012, 474), también se ha analizado la presencia de la información deportiva en la totalidad de las portadas de dichos rotativos durante este período (195 portadas).

Una vez recolectados los ejemplares que integran la muestra, se procedió al análisis cuantitativo y cualitativo del material utilizando el programa Microsoft Excel. Las categorías que han guiado el análisis del contenido son las siguientes: (1) Aparición en portada y extensión; (2) Deportes aparecidos en portada; (3) Día de publicación; (4) Menciones a disciplinas deportivas; y (5) Observaciones relevantes sobre la agenda deportiva (aspectos tratados, olvidados o tratados de forma superficial en la cobertura). 


\section{Resultados}

Atención limitada al deporte en portada

El análisis revela que los tres periódicos generalistas con más audiencia en España realizaron un seguimiento constante de la actualidad deportiva durante el Estado de alarma por la crisis sanitaria del Covid-19. No obstante, y debido a la importancia crucial de la información sanitaria y vinculada a los efectos sociales y económicos de la crisis, la presencia del deporte en las portadas fue limitada (Tabla 2). En la mayoría de los días analizados, el deporte estuvo ausente del espacio de portada: en 75,38\% de las portadas de El País (n=49), 61,54\% de las de El Mundo (n=40) y 73,85\% de las de $L a$ Vanguardia $(\mathrm{n}=48)$ no incluyeron menciones a la actualidad deportiva. Cuando los rotativos se hicieron eco del deporte, el espacio dedicado a este ámbito fue reducido (mayoritariamente, las noticias deportivas aparecieron en forma de titular o breve).

\begin{tabular}{c|c|c|c|c|c|c|}
\multirow{2}{*}{$\begin{array}{c}\text { Aparición en } \\
\text { portada }\end{array}$} & $\mathbf{n}$ & $\mathbf{2}$ & $\mathbf{2}$ & $\mathbf{2}$ & \multicolumn{2}{c|}{ El Ma Vanguardia } \\
\cline { 2 - 7 } & $\mathbf{n}$ & 75,38 & 40 & 61,54 & 48 & $\mathbf{0}$ \\
\hline $\begin{array}{c}\text { Sin aparición en } \\
\text { portada }\end{array}$ & 49 & 23,08 & 24 & 36,92 & 16 & 24,62 \\
\hline Titular o breve & 15 & 1,54 & 1 & 1,54 & 1 & 1,54 \\
\hline $\begin{array}{c}\text { Menos de media } \\
\text { página }\end{array}$ & 1 & 0,00 & 0 & 0,00 & 0 & 0,00 \\
\hline $\begin{array}{c}\text { Más de media } \\
\text { página }\end{array}$ & 0 & 100,00 & 65 & 100,00 & 65 & 100,00 \\
\hline Total & 65 &
\end{tabular}

Tabla 2. Aparición de la información deportiva en portada. Fuente: Elaboración propia.

En los tres rotativos el deporte más tratado en portada fue el fútbol, seguido de piezas centradas en múltiples disciplinas. Otros deportes presentados en portada fueron el baloncesto, el ciclismo, la Fórmula 1 y el tenis (Tabla 3).

\begin{tabular}{|c|c|c|c|c|c|c|}
\hline \multirow[b]{2}{*}{$\begin{array}{c}\text { Deporte en } \\
\text { portada }\end{array}$} & \multicolumn{2}{|c|}{ El País } & \multicolumn{2}{|c|}{ El Mundo } & \multicolumn{2}{|c|}{ La Vanguardia } \\
\hline & $\mathbf{n}$ & $\%$ & $\mathbf{n}$ & $\%$ & $\mathbf{n}$ & $\%$ \\
\hline Baloncesto & 0 & 0,00 & 1 & 4,00 & 0 & 0,00 \\
\hline Ciclismo & 1 & 6,25 & 0 & 0,00 & 0 & 0,00 \\
\hline Fórmula 1 & 1 & 6,25 & 2 & 8,00 & 1 & 5,88 \\
\hline Fútbol & 11 & 68,75 & 16 & 64,00 & 13 & 76,47 \\
\hline Polideportivo & 3 & 18,75 & 5 & 20,00 & 2 & 11,76 \\
\hline Tenis & 0 & 0,00 & 1 & 4,00 & 1 & 5,88 \\
\hline Total & 16 & 100,00 & 25 & 100,00 & 17 & 100,00 \\
\hline
\end{tabular}

Tabla 3. Agenda deportiva en portada. Fuente: Elaboración propia.

'Futbolización' de la información deportiva en tiempos de Covid-19

La cobertura informativa en las secciones de deportes se centró de forma prioritaria en el fútbol, que fue tratado en un total de 627 piezas (44,45\% de la muestra). Otros deportes con un fuerte seguimiento y arraigo en el contexto español, como el baloncesto, el tenis, el ciclismo, el atletismo o el balonmano fueron mencionados de forma regular durante la cobertura (Tabla 4). Por el contrario, otras disciplinas como la gimnasia, el waterpolo o el bádminton aparecieron de forma muy limitada en El País, El Mundo y La Vanguardia, demostrando que la situación de pandemia no modificó las prioridades informativas y las desigualdades preexistentes en la agenda deportiva convencional. 


\begin{tabular}{|c|c|c|c|c|}
\hline Deporte & El País & El Mundo & $\begin{array}{c}\text { La } \\
\text { Vanguardia }\end{array}$ & Total \\
\hline Atletismo & 21 & 6 & 28 & 55 \\
\hline Bádminton & 3 & 2 & 4 & 9 \\
\hline Baloncesto & 39 & 12 & 76 & 127 \\
\hline Balonmano & 14 & 4 & 33 & 51 \\
\hline Béisbol & 4 & 2 & 6 & 12 \\
\hline Boxeo & 6 & 1 & 8 & 15 \\
\hline Ciclismo & 28 & 4 & 27 & 59 \\
\hline Equitación & 2 & 0 & 0 & 2 \\
\hline Esgrima & 0 & 0 & 3 & 3 \\
\hline Fórmula 1 & 10 & 4 & 9 & 23 \\
\hline Fútbol & 230 & 86 & 311 & 627 \\
\hline Fútbol Sala & 7 & 2 & 12 & 21 \\
\hline Gimnasia & 10 & 1 & 8 & 19 \\
\hline Golf & 15 & 4 & 14 & 33 \\
\hline Halterofilia & 1 & 0 & 1 & 2 \\
\hline Hockey & 11 & 5 & 20 & 36 \\
\hline Judo & 1 & 1 & 0 & 2 \\
\hline Karate & 2 & 0 & 2 & 4 \\
\hline Motociclismo & 8 & 3 & 14 & 25 \\
\hline Natación & 16 & 3 & 15 & 34 \\
\hline Pelota vasca & 1 & 0 & 0 & 1 \\
\hline Piragüismo & 4 & 1 & 4 & 9 \\
\hline Remo & 2 & 0 & 1 & 3 \\
\hline Rugby & 3 & 3 & 16 & 22 \\
\hline Taekwondo & 3 & 0 & 0 & 3 \\
\hline Tenis & 25 & 10 & 39 & 74 \\
\hline Triatlón & 3 & 0 & 2 & 5 \\
\hline Voleibol & 3 & 1 & 2 & 6 \\
\hline Waterpolo & 4 & 3 & 6 & 13 \\
\hline
\end{tabular}

Tabla 4. Número de piezas donde se mencionaron los principales deportes en el contexto español. Fuente: Elaboración

Pese a la suspensión de las competiciones nacionales y continentales como la Liga de Campeones, la atención informativa sobre el fútbol fue extensiva a lo largo del período examinado. Uno de los ejes clave abordados en la cobertura fue la resolución de las principales ligas de fútbol europeas.

En relación a este punto, se han verificado dos hechos opuestos muy controvertidos. En primer lugar, la Ligue 1 francesa decidió parar el campeonato y otorgar el título al Paris Saint Germain. El combinado presidido por un fondo catarí tenía una diferencia abismal de puntos con respeto al segundo clasificado, en el momento en que la competición fue suspendida por la pandemia (Torres 2020a, 35). En segunda instancia, en Holanda también se decretó acabada la competición de fútbol. En el caso de la Eredivisie, a la llegada del periodo de confinamiento Ajax y AZ Alkmaar dominaban la clasificación con los mismos puntos, la única diferencia era una ventaja en el gol average de parte del club de Ámsterdam (Torres 2020b, 31). Pese a tratar estas cuestiones, faltaron en estos casos más análisis en profundidad, incluso con la ayuda de expertos sobre cómo estas decisiones pudieron sentar un precedente, ya que no quedó claro o por lo menos, no salió de los periódicos, cuáles fueron los criterios de una y otra liga para llegar a tomar las decisiones finales. Esta cuestión es particularmente relevante, puesto que estas sentencias han desatado demandas judiciales de parte de aquellos equipos que se han sentido injustamente o ilegalmente perjudicados por dichas disposiciones.

Los periódicos examinados también dedicaron una gran atención a la reanudación de la Bundesliga, cuyo regreso fue aprobado por el gobierno alemán a principios de mayo (Rodríguez 2020a, 42). "El experimento alemán" (Torres 2020c, 43) es un buen ejemplo de la cobertura realizada por los medios generalistas españoles, sin quizás preguntarse si el tema tenía realmente tanto interés. La Bundesliga es, como la abrumadora mayoría de las ligas nacionales de fútbol europeas, un campeonato en el que no existe pelea real para el título, como queda demostrado por 
los resultados de los últimos lustros. Además, cuesta entender, siendo la Bundesliga una competición que apenas encuentra espacio en los periódicos españoles, cómo de repente se transformó en un evento digno de tanta atención, más allá de la valía de las piezas en el intento de reflexionar sobre cómo se volvería a la normalidad en el resto de países. Más que pensar en cuándo se reanudaría dicho campeonato, faltaron voces críticas, capaces de pensar en fórmulas nuevas para aumentar el interés de estas competiciones domésticas.

Esta atención entra en contraste con el escaso peso específico que los medios dedicaron a la afectación de la crisis sobre las selecciones nacionales de fútbol. De hecho, la noticia del aplazamiento inmediato de la Eurocopa se reportó en todos los periódicos sin ulteriores análisis. Es llamativo destacar cómo un evento muy importante, que junto con el Mundial y la Liga de Campeones forma parte de las grandes competiciones del fútbol, se aplazó tras una reunión muy rápida a la vez que unas competiciones mucho menos relevantes como las ligas nacionales generaron intensas batallas en el intento de salvarlas, como se reportó en el artículo "Goles en silencio" (Martínez 2020a, 60).

Lógicamente, la afectación de la crisis del Covid-19 sobre la liga de fútbol española acaparó gran parte de la cobertura en los tres periódicos analizados. Se abordaron cuestiones de primer orden como el enfrentamiento entre la Asociación de Futbolistas Españoles (AFE) y la Liga de Fútbol Profesional (LFP) por los ERTE (Novo 2020, 48), la afectación de la crisis sobre los derechos televisivos del fútbol (Calonge 2020a, 46) o los problemas jurídicos de los jugadores sin contrato (Lidón 2020, 35). En paralelo, se arrojó luz sobre las opiniones de los jugadores sobre el riesgo de reemprender la competición (La Vanguardia 2020a, 41). La polémica sobre los test masivos a los futbolistas también recibió una atención preferente (Arribas 2020a, 31), aunque los periódicos no albergaron debates profundos sobre la necesidad real de realizar dichos test. En un primer momento, los diarios criticaron mayoritariamente la oportunidad de hacerlo, críticas que retomaron muchos personajes públicos. De repente, sin embargo, esta tendencia cambió y se empezó a testar los futbolistas sin apenas distinguirse en la prensa algún análisis crítico, excepto en la pieza "Test y regreso por fases: así volverá La Liga a entrenar" (El País 2020, 43). Ninguno de los tres medios consultados profundizó en el hecho de que a los hombres se les hicieran test para saber si tenían el virus y a las mujeres deportistas, no.

Cabe resaltar que los medios analizados también cubrieron las muertes, a causa del Covid-19, de Lorenzo Sanz, ex presidente del Real Madrid, y de Radomir Antić, ex entrenador entre otros del Atlético de Madrid, Real Madrid y F.C. Barcelona. A raíz de la muerte de Sanz, se habló de la hazaña del Real Madrid en la Liga de Campeones a través de piezas como "Lorenzo Sanz, el presidente de la Séptima" (Toro 2020, 86). Sin embargo, se echó en falta un análisis más en profundidad de la temporada en la Liga de Campeones del Atlético (1996-97), tras el doblete conseguido por Antić en la temporada anterior. Más allá de estos fallecimientos a causa del Covid-19, El País, El Mundo y La Vanguardia se hicieron eco a través de múltiples piezas de la muerte y del legado futbolístico y comunicativo de Michael Robinson (Martínez 2020b, 40).

Pese a la atención constante sobre el fútbol masculino, el tratamiento del impacto de la pandemia sobre el fútbol femenino fue muy reducido, en clara consonancia con la escasa cobertura que reciben las mujeres deportistas en todos los contextos y espacios informativos. Los periódicos no analizaron en profundidad la gran disparidad que se produjo entre el fútbol femenino y el fútbol masculino, así como las desmedidas ventajas que el mismo fútbol masculino ha tenido con respeto a todos los demás deportes en España. A lo largo de los últimos años, los medios de comunicación han abanderado una igualdad entre el fútbol masculino y femenino. No obstante, al decretarse el estado de alarma, los medios respaldaron el discurso que la liga de fútbol masculina se debía terminar como fuera, mientras que acríticamente se aceptó que la liga femenina (Liga Iberdrola) no debía reemprenderse al sentenciarse el FG Barcelona como el campeón. Los periódicos apenas albergaron debates sobre este tema. Una excepción fue la pieza de El País "Más pleitos que goles en la Liga femenina" (Calonge 2020b, 46). Otras noticias relevantes sobre fútbol femenino, como el aplazamiento de la Eurocopa femenina a 2020 (Gómez 2020a, 34) o el hecho que un juez federal 
rechazara la igualdad salarial de las futbolistas en EE.UU. (La Vanguardia 2020b, 62) fueron reportadas de forma breve y principalmente a través de información de agencia.

\section{Desigualdades en la agenda polideportiva}

Siguiendo el mismo patrón que en la cobertura futbolística, gran parte de la información polideportiva se centró en la afectación de la crisis sobre las principales competiciones deportivas a nivel global. Entre otras cuestiones de primer orden, se trató el aplazamiento de los Juegos Olímpicos y Paralímpicos de Tokio 2020 a 2021 (Arribas, 2020b, 35), la cancelación del Campeonato de Wimbledon de tenis (Heredia 2020a, 42) y la reorganización en el calendario de las tres grandes citas del ciclismo mundial: el Tour de Francia, el Giro de Italia y La Vuelta a España (Arribas 2020c, 34; García Luque 2020, 43). Después de informar sobre las rebajas salariales de los jugadores de la NBA (Álvarez 2020a, 37), la prensa generalista española también ahondó en cómo se volvería a reanudar la temporada de la competición de baloncesto estadounidense. Finalmente, el final de la competición se disputaría en Orlando, capaz de disponer de tres pabellones de medianas dimensiones en un área muy restringida. La decisión de la NBA (semejante al caso de la Liga Endesa en España), se yuxtapone al anuncio de la liga europea de baloncesto, la Euroliga, que declaró cancelada su temporada al no tener las garantías de poder organizar el traslado de todos los equipos en una hipotética sede única. Además de estos desenlaces, los medios examinados también se hicieron eco del aplazamiento por un año del Eurobasket masculino, originalmente previsto para 2021 (Dotras 2020, 49).

Durante las primeras semanas del estado de alarma, los periódicos trataron de arrojar luz sobre los retos que para atletas como Salma Paralluelo o Jesús García Bragado conllevó la imposibilidad de entrenar en instalaciones deportivas y centros de tecnificación (Arribas 2020d, 45; Calonge 2020c, 37). Tampoco se rehuyó de hablar de la afectación económica de la pandemia sobre el balonmano español y sobre los tenistas más perjudicados por el parón de las competiciones (Calonge 2020d, 36; Ciriza 2020, 37). Sin embargo, poco espacio tuvieron ciertas contradicciones que aparecieron a medida que se iban paulatinamente frenando las restricciones. Por ejemplo, mientras que fueron posible los entrenamientos de equipos de fútbol, campeonas olímpicas de la talla de Mireia Belmonte, Carolina Marín o Lydia Valentín -las tres curiosamente practicando deportes que no prevén contacto con una adversaria- tuvieron que quedarse en casa. La atención a esta cuestión fue mínima: se publicó una pieza por cada periódico analizado, como por ejemplo "Hay diferencias injustas" (Heredia 2020b, 41). Estas desigualdades son particularmente graves, considerando que se tratan de atletas capaces de cosechar medallas de oros en los Juegos Olímpicos, siendo por lo tanto entre las máximas representantes de España en el mundo.

Durante el período de pandemia, otros deportes como la fórmula 1, la gimnasia, el golf o el waterpolo recibieron una atención discreta por parte de los rotativos examinados (La Vanguardia 2020c, 49; Morenilla 2020, 37; Rodríguez 2020b, 42). Otros deportes minoritarios, como la pelota vasca, fueron mencionados de forma puntual (Rivas 2020, 36). Sin embargo, otras muchas disciplinas fueron minimizadas en la cobertura. La infrarrepresentación del deporte adaptado resulta notoria: únicamente 15 piezas $(1,06 \%$ de la muestra) se hicieron eco de la cancelación de los Juegos Paralímpicos de Tokio 2020 y de la afectación de dicha cancelación sobre sus participantes. Aún así, esta información no se trató de forma independiente sino siempre en relación con la suspensión de los Juegos Olímpicos.

Se encontró un discreto número de artículos en que los expertos analizaron cómo se podrían reanudar las competiciones, pero aquí también la gran mayoría se concentró solo en fútbol, y sobre todo en ligas nacionales de futbol. Se echó en falta un análisis más amplio sobre los riesgos de volver a practicar el deporte con una mirada más amplia considerando las diferentes disciplinas y las distintas modalidades de cada certamen. Faltaron investigaciones en profundidad sobre cómo todos los deportes estaban moviéndose en el intento de volver a una nueva normalidad. Es lícito pensar que una competición que supone una lucha entre dos contrincantes, como el judo o el boxeo, o un deporte que presupone un continuo contacto entre varios jugadores pueden resultar mucho más 
peligrosas de retomar con respecto, por ejemplo, a un evento de tenis individual o en cualquier otra disciplina en la que no se producen contactos entre los atletas.

Estas carencias referentes a la información polideportiva contrastan con la atención que desde los rotativos españoles se dedicó a aspectos superficiales y sensacionalistas. Durante el período analizado se asistió a una banalización del periodismo deportivo a través de relatos sobre infinidad deportistas que se entrenaban en sus propias residencias. En muchos casos más que entrenos serios eran unos momentos más recreativos que de verdadero entrenamiento. En este aspecto, el periodismo de la prensa escrita siguió, aunque en manera menor, la estela dejada por muchos noticieros de televisión. Raras fueron las entrevistas de calidad sobre la vida de los deportistas durante el confinamiento. Una de las más destacadas fue "Lydia Valentín: 'hay que ser mentalmente fuerte para lo que vendrá" (Álvarez 2020b, 35), publicada en El País.

También en relación a la banalización de los contenidos deportivos, la prensa ahondó en detalles triviales y curiosidades como el coche de lujo que Cristiano Ronaldo regaló a su madre (La Vanguardia 2020d, 42) o el gasto que las universidades y clubes en Estados Unidos realizan para diseñar y entrenar a sus mascotas (La Vanguardia 2020e, 42). Historias controvertidas, como el declive y prisión de Ronaldinho (Pires 2020, 38) o las acusaciones de corrupción en el F.C. Barcelona (Ruipérez 2020, 40) también resonaron en la cobertura de los medios analizados, en especial en La Vanguardia. También se incluyeron varios artículos sobre la serie documental The Last Dance, que pretende analizar el último año en los Chicago Bulls de Michael Jordan y que emitió una plataforma de contenidos de pago. El producto es poco periodístico, pese a ser un tema muy relevante de la historia del baloncesto. Los periódicos más que bucear y analizar a fondo en los temas deportivos que podía proponer dicho documental, dedicaron su atención a hablar de si un jugador era antipático, si hubo peleas de vestuario entre atletas y de otros aspectos parecidos más apropiados para otro tipo de secciones.

\section{Discusión y conclusiones}

La presente investigación ha examinado la cobertura deportiva llevada a cabo por El País, El Mundo y La Vanguardia durante los primeros 65 días del estado de alarma para determinar si la coyuntura del Covid-19 ofreció una oportunidad para diversificar la agenda deportiva y transcender las limitaciones preexistentes en esta área de especialización.

Los resultados del estudio revelan que la prensa generalista española no aprovechó la enorme oportunidad de confeccionar un producto periodístico de calidad en la situación de emergencia causada por el Covid-19. No se articuló una cobertura deportiva distintiva que transcendiera la uniformidad temática del periodismo deportivo convencional y que abogara por fomentar la diversidad en un sentido amplio (Ramon-Vegas et al., 2020; Rojas-Torrijos, 2012; Sainz-deBaranda, Barbero-González y Fernández-Fernández, 2019).

Conscientes de la gran capacidad de atracción del deporte en un momento de dificultades económicas y de fragmentación de las audiencias (Rojas-Torrijos 2018; Serazio 2019), los medios españoles siguieron apostando por un tratamiento extensivo del deporte durante la coyuntura de crisis. Este hallazgo es consistente con la situación desarrollada en múltiples países y contextos periodísticos. Como señala Hutchins (2020), "el deporte en tiempos de Covid-19 presenta una paradoja. No hay partidos, resultados, momentos clave y controversias en el terreno de juego de las que hablar [...] sin embargo, a pesar de que se multiplican las historias de muertes e infecciones, las noticias y la conversación en línea sobre el deporte que envuelve las ligas y eventos más populares no menguan".

En un escenario sin competiciones, la atención mediática sobre el fútbol masculino y profesional permaneció intacta. En la búsqueda permanente del capital económico (Bourdieu 1998; English 2016), la prensa maximizó la información sobre las grandes ligas y clubes de fútbol. Si bien otros deportes con gran seguimiento en España como el baloncesto, el tenis, el ciclismo, el atletismo o el balonmano aparecieron de forma recurrente en la cobertura, se limitó la visibilidad de otros muchos deportes y protagonistas que permanecen en la periferia del complejo mediático. De esta forma, la 
pandemia no representó un momento de ruptura sino de continuidad en el tratamiento deportivo: la crisis del Covid-19 no modificó las prioridades existentes, sino que intensificó la atención sobre aquellos deportes que ya acumulaban más poder mediático.

La cancelación de los Juegos Olímpicos y Paralímpicos de Tokio 2020 ofreció la oportunidad de reflexionar sobre el impacto del Covid-19 en muchas otras disciplinas, pero la noticia no fue un catalizador para realizar un seguimiento posterior sobre éstas de una forma sostenida. Gracias a la ausencia de las grandes competiciones, las secciones deportivas, siempre bastante ajenas a los deportes minoritarios, tenían una buena oportunidad para acercar el lector a dichas disciplinas. Si bien se abordaron de forma puntual las consecuencias de la crisis sobre el deporte femenino, la atención por parte de la prensa generalista no fue suficiente para transcender la asimetría estructural de género que se produce en el periodismo deportivo (Franks y O’Neill 2016; Gutiérrez San Miguel 2016; Herrero-Gutiérrez 2018; Sainz-de-Baranda 2014). Pese a la cancelación de los Juegos Paralímpicos, la atención sobre los deportistas adaptados fue prácticamente nula, reforzando de esta forma su invisibilidad en la esfera mediática (Solves et al. 2019).

Estas disparidades en la agenda conllevan repercusiones sociales, económicas y deportivas de primer orden. De forma inequívoca, el fútbol es el deporte más seguido y consumido a nivel mediático en el contexto español (Llopis-Goig 2014). Sin embargo, parte de la responsabilidad y función social de los medios de comunicación es velar por fomentar una cultura deportiva diversa, que ayude a ampliar los horizontes y referentes de los ciudadanos. A raíz de la crisis del Covid-19, multitud de deportes que ya contaban con recursos económicos limitados (como por ejemplo el balonmano, el waterpolo o el hockey) han quedado desprovistos de sus principales fuentes de ingresos: la venta de entradas, los patrocinios y los ingresos por las retransmisiones televisivas (Horky 2021). En un escenario complejo donde la estabilidad financiera de los deportes minoritarios está en juego, no conviene olvidar que la atención por parte de los medios generalistas y especializados resulta fundamental para preservar su visibilidad social.

Es cierto que los medios generalistas realizaron una labor intensiva para conectar el deporte con cuestiones sanitarias, sociales, económicas, políticas y jurídicas de primer orden, desmarcándose así de la noción que los medios prestan una limitada atención al larger framework, esto es, a las cuestiones contextuales relevantes más allá del terreno de juego (McEnnis 2020; Serazio 2019). Sin embargo, también se detectó en múltiples instancias una atención innecesaria sobre cuestiones sensacionalistas o triviales. En relación a este punto, no conviene olvidar que "ante el gran flujo de información que llega a los ciudadanos a través de múltiples canales, los medios pugnan por la visibilidad de sus contenidos, lo que con frecuencia se consigue a través de contenidos impactantes" (Masip et al. 2020, 8).

Dicho esto, la atención sobre algunas cuestiones anecdóticas podría haberse aprovechado para: (1) ahondar más en otros deportes; (2) aportar una visión crítica sobre el estatus quo del deporte, en pos de intentar mejorar ciertos aspectos organizativos de los grandes eventos y resaltar las contradicciones entre múltiples disciplinas y (3) dotar de una mayor profundidad al abordaje histórico del deporte, atendiendo a un mayor espectro de modalidades y protagonistas. Los medios también podrían haber aprovechado la oportunidad de acercar al lector a los deportes más tradicionalmente norteamericanos, que cuentan con millones seguidores en todo el mundo, así como tratar cuestiones de gran relevancia que sucedieron en otros continentes como Asia o América del Sur. A través de estos ingredientes, los periódicos hubiesen podido ofrecer un papel didáctico que hubiese representado una apuesta ganadora de cara al futuro a medio y largo plazo. A continuación, se destacan tres aspectos de especial relevancia que se podrían haber desarrollado.

En primer lugar, considerando la escasa presencia de eventos en directo, otros temas posibles que hubiesen podido enriquecer las secciones deportivas de los periódicos son los aniversarios de personas relevantes del mundo del deporte y recurrencias de grandes competiciones y eventos del pasado. Con el recurso periodístico de ir a buscar fechas importantes en el calendario se hubiese podido hacer un viaje en distintos aspectos de la carrera de grandes deportistas o analizar un duelo histórico con el valor añadido de la mirada actual. No se encontró rastro por ejemplo del cumpleaños número 50 de dos grandes tenistas: Andre Agassi, mito del tenis y gran rival durante los 
míticos 90 de Pete Sampras, y Gabriela Sabatini, campeona del Abierto de los Estados Unidos en 1990 y finalista de Wimbledon. También pasó inadvertido el aniversario número 60 de Franco Baresi, capitán de la época más gloriosa del Milán.

También en relación a la perspectiva histórica en la cobertura deportiva, se señala un caso notorio que presentó el diario El País. Normalmente los lunes se publica la sección de Alfredo Relaño, titulada "Memorias en blanco y negro", en la que el periodista relata una historia deportiva del pasado. Curiosamente a lo largo de un mes dicha sección se suspendió para reaparecer nuevamente. El mismo periódico creó una sección diaria llamada "El jugador que deslumbró a...", en la que proponía una pieza en la que un gran jugador hablaba de su propio ídolo del pasado. En este marco, se ofreció al lector la posibilidad de ahondar en una gran variedad de protagonistas, entre ellos los futbolistas argentinos Diego Armando Maradona (Ortego 2020, 40) y Mario Kempes (Álvarez 2020c, 37) o el ciclista francés Bernard Hinault (Arribas 2020e, 38). Sin embargo, se podría haber aprovechado dicha sección para ampliar el espectro de deportes tratados.

En segundo lugar, varias noticias de gran relevancia sobre la National Football League (NFL) recibieron una atención muy discreta por parte de la prensa española. Una de estas informaciones hace referencia al "traslado" de Tom Brady, seis veces campeón de la Super Bowl. Tras 20 años representando a los New England Patriots, el quarterback tomó la decisión de dejar la ciudad de Boston y el gurú de los banquillos Bill Belichick, para trasladarse a los Tampa Bay Buccaneers. Brady y Belichick representan a la pareja más ganadora en la historia de dicho deporte y gracias a ellos la franquicia del noreste de los Estados Unidos es la que ostenta más títulos en la historia de la NFL, junto a los Steelers de Pittsburgh. La noticia fue comentada en todas las secciones deportivas de los principales periódicos de Norteamérica y de los demás continentes, Europa incluida. Únicamente La Vanguardia se hizo eco de la noticia a través de un breve, titulado "Brady rompe con su historia" (La Vanguardia 2020f, 41).

Otra noticia mayúscula en relación a la NFL fue que el draft del año 2020 se realizó por videoconferencia debido al Covid-19. La selección de los nuevos jugadores provenientes de las universidades se llevó a cabo por conexión remota y con un éxito de aficionados delante de las pantallas. La prensa española mencionó lo ocurrido, pero sin ningún tipo de análisis. La pieza "Un draft desde el sótano de casa" (Gómez 2020b, 32) es un buen ejemplo de cómo se habló del evento, donde simplemente se señaló cómo puede ser un modelo para el draft de la NBA, una liga seguida ampliamente por la prensa española. Sin embargo, no se habló de ninguno de los seleccionados, algunos de ellos ya con interesantes historias en sus mochilas.

En tercera instancia, la prensa no realizó un seguimiento exhaustivo de dónde había deporte en directo para ver qué sucedía. Tras un mes, correspondiente con las primeras semanas de encierro, en el que realmente no se desarrollaron eventos deportivos en directo, con la única excepción del campeonato de fútbol de Bielorrusia, certamen de muy bajo perfil, se empezaron a jugar algunos campeonatos muy relevantes. En concreto se reanudaron el campeonato de béisbol de Taiwán y, más tarde, el de Corea del Sur. Ambas son ligas de primerísimo nivel que representan a dos países capaces de alcanzar la cumbre mundial. Taiwán llegó la final en los Juegos Olímpicos de Barcelona 1992. Perdió en Hospitalet de Llobregat con Cuba, que curiosamente cayó en la final de Pekín 2008 contra Corea del Sur. Ante la imposibilidad de acudir presencialmente adonde sí había competición, hubiera sido interesante emplear informaciones de agencia y contar con las voces de corresponsales y expertos para analizar cómo afrontan allí las cuestiones relacionadas con la pandemia y el distanciamiento social.

A raíz de todas estas consideraciones, el periodismo deportivo en tiempos del Covid-19 se enfrenta al reto clave de trabajar para fomentar una mayor cultura deportiva: transcender el localismo, ampliar el menú deportivo y proporcionar ángulos alternativos a los deportes destacados, superando la "redundancia, uniformidad y similitud que tan frecuentemente se puede encontrar en los contenidos deportivos vinculados a la estricta actualidad" (Ramon-Vegas y Tulloch 2016, 407). En su práctica periodística, el periodista también deberá acostumbrarse a trabajar sin público. En el relato de las crónicas sin público, no se presentará la opción de relatar lo que ocurre en la grada. Por el contrario, sí se podrán captar mucho más fácilmente palabras pronunciadas por los deportistas, la 
comunicación entre ellos, entre un competidor y un entrenador o un colegiado, entre otras cuestiones. Sin embargo, para interpretar las competiciones, el periodista deberá ir más allá del terreno de juego para aportar contexto y valor añadido a su tarea.

La presente investigación se ha centrado en analizar la cobertura deportiva realizada durante los 65 primeros días del estado de alarma por los tres medios generalistas con más audiencia en España. Pese a las limitaciones derivadas del período temporal de la observación, la aproximación realizada es un punto de partida para seguir monitorizando esta área de especialización. Partiendo de los hallazgos de este trabajo, futuros estudios deberán radiografiar de qué forma los medios examinados, los periódicos de información deportiva en España y otras organizaciones públicas y privadas en diferentes países y culturas periodísticas siguen abordando el deporte en tiempos del Covid-19. Trabajos futuros también deberán examinar otros medios de información general con larga trayectoria y tirada a nivel nacional, como $A B C$, así como otros rotativos regionales de gran calado como El Correo y La Voz de Galicia.

El análisis del contenido podría triangularse con entrevistas en profundidad o grupos de discusión con periodistas y editores, que permitirían obtener un conocimiento más profundo sobre los criterios informativos, los valores y los recursos económicos y temporales disponibles en los medios de comunicación. No conviene olvidar que en el escenario actual, la escasez de tiempo y de recursos económicos en las redacciones tienen una clara incidencia sobre el tipo de periodismo deportivo que se desarrolla y las cuestiones que pueden abordarse (Broussard 2020). Esta aproximación cualitativa también permitiría conocer de forma pormenorizada las limitaciones y retos principales a los que se están enfrentado los profesionales de la información deportiva durante la pandemia, entre ellos la movilidad limitada de los trabajadores y la consiguiente agudización de las dificultades de acceso a la información. Este conocimiento más pormenorizado permitirá ahondar en la forma en que estos condicionantes y dificultades laborales se trasladan a los contenidos periodísticos.

\section{Referencias bibliográficas}

AIMC. 2020. "Marco General de los Medios en España 2020", Asociación para la Investigación de Medios de Comunicación, https://bit.ly/30OCEEn (consultado el 10 julio 2020).

Altheide, David L. 1996. Qualitative media analysis. Thousand Oaks, California: Sage.

Álvarez, Robert. 2020a, 4 abril. "La NBA reduce un 25\% el sueldo de los jugadores", El País, 37.

Álvarez, Robert. 2020b, 11 abril. "Lydia Valentín: 'hay que ser mentalmente fuerte para lo que vendrá", El País, 35.

Álvarez, Robert. 2020c, 11 mayo. "Me enganché al fútbol con la idea de ser Mario Kempes", El País, 37.

Arribas, Carlos. 2020a, 30 abril. "El fútbol espera el permiso de Sanidad para los test", El País, 31.

Arribas, Carlos. 2020b, 24 marzo. "Objetivo Tokio 2021”, El País, 35.

Arribas, Carlos. 2020c, 6 mayo. "Todo el ciclismo del año, comprimido en 100 días", El País, 34.

Arribas, Carlos. 2020d, 15 marzo. "García Bragado, de Sant Gugat a una cueva en Guadix", El País, 45.

Arribas, Carlos. 2020e, 9 mayo. "Quién hubiera tenido el motor de Hinault, y ese amor por la gesta", El País, 38.

Bourdieu, Pierre. 1998. On television and journalism. Londres: Pluto Press.

Boyle, Raymond. 2017. "Sports journalism: Changing journalism practice and digital media". Digital Fournalism 5, n. ${ }^{\circ}$ 5: 493-5. https://doi.org/10.1080/21670811.2017.1281603

Broussard, Ryan. 2020. "'Stick to Sports' is Gone: A Field Theory. Analysis of Sports Journalists' Coverage of Socio-political Issues". Fournalism Studies 21, n. ${ }^{\circ}$ 12: 1627-43. https://doi.org/ 10.1080/1461670X.2020.1785323 
Bryman, Alan. 2016. Social research methods. Oxford: Oxford University Press.

Calonge, Lorenzo. 2020a, 10 mayo. "El nuevo melón de la batalla televisiva". El País, 46.

Calonge, Lorenzo. 2020b, 10 mayo. "Más pleitos que goles en la Liga femenina". El País, 46.

Calonge, Lorenzo. 2020c, 27 abril. "El salto de sillas de Salma Paralluelo". El País, 37.

Calonge, Lorenzo. 2020d, 13 abril. "El balonmano español, en ERTE”. El País, 36.

Calvo-Ortega, Elena y Begoña Gutiérrez San Miguel. 2016. "La mujer deportista y periodista en los informativos deportivos de televisión. Un análisis comparativo con respecto a su homólogo masculino". Revista Latina de Comunicación Social, n. ${ }^{\circ}$ 71: 1230-42. https://doi.org/10.4185/ RLCS-2016-1143

Carvajal, Miguel. 2020, 11 mayo. "El impacto del COVID-19 en la economía del periodismo". Blog Noticias y pistas del Máster en Innovación en Periodismo. https://bit.ly/3vRyIyK (consultado el 16 de junio 2021).

Casero-Ripollés, Andreu. 2020. "Impact of Covid-19 on the media system. Communicative and democratic consequences of news consumption during the outbreak". El profesional de la información 29, n. ${ }^{\circ}$ 2: 1-11. https://doi.org/10.3145/epi.2020.mar.23

Ciriza, Alejandro. 2020, 19 abril. "El tenis crea un fondo de ayuda a sus 'autónomos"'. El País, 37.

Dotras, Oriol. 2020, 11 abril. "El Eurobasket masculino se retrasa un año". La Vanguardia, 49.

El País. 2020, 5 abril. "Test y regreso por fases: así volverá La Liga a entrenar.” El País, 43.

English, Peter. 2014. "The same old stories: Exclusive news and uniformity of content in sports coverage". International Fournal of Sports Communication 7, n. ${ }^{\circ}$ 4: 477-94. https://doi.org/ 10.1123/IJSG.2014-0026

English, Peter. 2016. "Mapping the sports journalism field: Bourdieu and broadsheet newsrooms". fournalism 17, n. ${ }^{\circ}$ 8: 1001-17. https://doi.org/10.1177/1464884915576728

English, Peter. 2017. "Cheerleaders or critics? Australian and Indian sports journalists in the contemporary age". Digital Fournalism 5, n. ${ }^{\circ}$ 5: 532-48. https://doi.org/ $10.1080 / 21670811.2016 .1209082$

España, 2020. "Real decreto 463/2020, de 14 de marzo, por el que se declara el estado de alarma para la gestión de la situación de crisis sanitaria ocasionada por el Covid-19". Boletín Oficial del Estado, n. ${ }^{\circ}$ 67: 25390-400. https://www.boe.es/eli/es/rd/2020/03/14/463

Franks, Suzanne y Deirdre O’Neill. 2016. "Women reporting sport: Still a man's game?” Fournalism 17, n. ${ }^{\circ}$ 4: 474-92. https://doi.org/10.1177/1464884914561573

García Luque, Xavier. 2020, 6 mayo. "El ciclismo del coronavirus". La Vanguardia, 43.

Gómez, Daniel. 2020a, 24 abril. "La Eurocopa femenina se aplaza a 2022”. El País, 34.

Gómez, Daniel. 2020b, 25 abril. "Un draft desde el sótano de casa”. El País, 32.

Heredia, Sergio. 2020a, 2 abril. "Wimbledon cerrará la puerta". La Vanguardia, 42.

Heredia, Sergio. 2020b, 13 mayo. "Hay diferencias injustas". La Vanguardia, 41.

Herrero-Gutiérrez, Francisco Javier. 2018. "Los periódicos deportivos españoles. Análisis comparativo de la noticia principal de portada en los diarios Marca, As, Mundo Deportivo y Sport". Estudios sobre el Mensaje Periodístico 24, n. ${ }^{\circ}$ 2: 1353-65. https://doi.org/10.5209/ ESMP.62221

Horky, Thomas. 2021. "No sports, no spectators - no media, no money? The importance of spectators and broadcasting for professional sports during COVID-19". Soccer E Society 22, n. ${ }^{\circ}$ 1-2: 96-102. https://doi.org/10.1080/14660970.2020.1790358

Hutchins, Brett. 2020, 17 abril. "COVID-19: Despite its flaws, sport's significance runs deep in society", Lens, https://bit.ly/2PGPMF8 (consultado el 10 julio 2020). 
La Vanguardia. 2020a, 6 mayo. "La plantilla del Eibar se posiciona: Tenemos miedo". La Vanguardia, 41.

La Vanguardia. 2020b, 3 mayo. "El juez rechaza la igualdad salarial de las futbolistas de EE.UU". La Vanguardia, 62.

La Vanguardia. 2020c, 4 mayo. "El Sabadell disiente del acuerdo liguero". La Vanguardia, 49.

La Vanguardia. 2020d, 4 mayo. "Coche de lujo para la madre de Cristiano". La Vanguardia, 42.

La Vanguardia. 2020e, 14 abril. "Las universidades y clubs profesionales de Estados Unidos gastan millones en diseñar (y entrenar si es necesario) la mascota perfecta". La Vanguardia, 42.

La Vanguardia. 2020f, 18 marzo. "Brady rompe con su historia". La Vanguardia, 41.

Lázaro-Rodríguez, Pedro y Enrique Herrera-Viedma. 2020. "Noticias sobre Covid-19 y 2019nCoV en medios de comunicación de España: el papel de los medios digitales en tiempos de confinamiento". El profesional de la información 29, n. ${ }^{\circ}$ 3: 1-11. https://doi.org/10.3145/ epi.2020.may.02

Lidón, Inma. 2020, 9 abril. "El limbo jurídico de los jugadores sin contrato". El Mundo, 35.

Llopis-Goig, Ramon. 2014. "The predominance of soccer in the sport and leisure habits of Spanish society". Sport in Society 17, n. 6: 824-41. https://doi.org/10.1080/17430437.2014.882909

López-Aranguren, Eduardo. 2016. "El análisis de contenido tradicional”. En El análisis de la realidad social: métodos y técnicas de investigación, editado por Manuel García Ferrando, Francisco Ricardo Alvira, Luis Enrique Alonso y Modesto Escobar, 594-616. Madrid: Alianza Editorial.

López-Rabadán, Pablo y Andreu Casero-Ripollés. 2012. "La evolución de la agenda mediática española (1980-2010). Un análisis longitudinal de la portada de la prensa de referencia". Revista Latina de Comunicación Social, n. ${ }^{\circ}$ 67: 470-93. https://doi.org/10.4185/RLCS-2012-964

Martínez, Juan Bautista. 2020a, 17 mayo. "Goles en silencio". La Vanguardia, 60.

Martínez, Juan Bautista. 2020b, 29 abril. "Un genio con acento". La Vanguardia, 40.

Mena, Francisco. 2020, 9 mayo. "El mapa del coronavirus en el deporte: más de 250 suspensiones o cancelaciones", Rtve.es, https://bit.ly/2PK7EyL (consultado el 10 julio 2020).

Márquez-Ramírez, Mireya y José Luis Rojas-Torrijos. 2017. "¿Periodismo deportivo pasivo o proactivo? La cobertura del FIFAGate en la prensa deportiva de México y España”. Cuadernos.info, n. ${ }^{\circ}$ 40: 173-88. https://doi.org/10.7764/cdi.40.1009

Masip, Pere, Sue Aran-Ramspott, Carlos Ruiz-Caballero, Jaume Suau, Ester Almenar y David Puertas-Graell. 2020. "Consumo informativo y cobertura mediática durante el confinamiento por el Covid-19: sobreinformación, sesgo ideológico y sensacionalismo". El profesional de la información 29, n. ${ }^{\circ}$ 3: 1-12. https://doi.org/10.3145/epi.2020.may. 12

McCombs, Maxwell. 2004. Setting the agenda. The mass media and public opinion. Cambridge: Polity Press.

McCombs, Maxwell y Donald Shaw. 1972. "The agenda-setting function of mass media". Public Opinion Quarterly 36, n. ${ }^{\circ}$ 2: 176-87. https://doi.org/10.1086/267990

McEnnis, Simon. 2020. "Toy department within the toy department? Online sports journalists and professional legitimacy". Fournalism 21, n. ${ }^{\circ}$ 10: 1415-31. https://doi.org/ $10.1177 / 1464884918797613$

Montero-Ramos, Francisco Javier. 2017. "Deontología del periodismo deportivo. Principios fundamentales y tratamiento en prensa". Tesis doctoral, Universidad Complutense de Madrid.

Morenilla, Juan. 2020, 15 mayo. "El protocolo del golf: los 'caddies' no tocan el palo". El País, 37.

Novo, Carlos. 2020, 4 abril. "La Liga rompe con la AFE". La Vanguardia, 48. 
O’Neill, Deirdre y Matt Mulready. “The Invisible Woman? A comparative study of women's sports coverage in the UK national press before and after the 2012 Olympic Games". Fournalism Practice 9, n. ${ }^{\circ}$ 5: 651-68. https://doi.org/10.1080/17512786.2014.965925

Ortego, Enrique. 2020, 3 mayo. "Que últimos 10 metros de Maradona". El País, 40.

Parnell, Daniel, Paul Widdop, Alex Bond y Rob Wilson. 2020. "Covid-19, networks and sport". Managing Sport and Leisure, 1-7. https://doi.org/10.1080/23750472.2020.1750100

Pires, Breiller. 2020, 16 marzo. "Traición, declive y prisión, la última década de Ronaldinho". El País, 38.

Pulido, Cristina, Beatriz Villarejo-Carballido, Gisela Redondo-Sama y Aitor Gómez. 2020. "Covid-19 infodemic: More retweets for science-based information on coronavirus than for false information". International Sociology 35, n. ${ }^{\circ} 4:$ 377-92. https://doi.org/ $10.1177 / 0268580920914755$

Ramon-Vegas, Xavier, Eva Gómez-Colell, Mónica Figueras-Maz y Pilar Medina-Bravo. 2020. “Las mujeres como outsiders en el periodismo deportivo: percepción de las estudiantes y personas expertas". Estudios sobre el Mensaje Periodístico 26, n. .o 3: 1183-94. https://doi.org/10.5209/ esmp. 64526

Ramon-Vegas, Xavier y Christopher Tulloch. 2016. "Periodismo deportivo de largo formato en la era digital". El profesional de la información 25, n. ${ }^{\circ}$ 3: 404-12. https://doi.org/10.3145/ epi.2016.may. 10

Ramon-Vegas, Xavier y Christopher Tulloch. 2021. "Life beyond clickbait journalism: A transnational study of the independent football magazine market". Communication \& Sport 9, n. ${ }^{\circ}$ 4: 603-34. https://doi.org/10.1177/2167479519878674

Rivas, Jon. 2020, 6 abril. "La cesta punta remata el cierre del deporte en EEUU”. El País, 36.

Rodero, Emma. 2020. "La radio: el medio que mejor se comporta en las crisis. Hábitos de escucha, consumo y percepción de los oyentes de radio durante el confinamiento por el Covid-19". El profesional de la información 29, n. ${ }^{\circ}$ 3: 1-15. https://doi.org/10.3145/epi.2020.may.06

Rodríguez, Roberto. 2020a, 7 mayo. "Merkel aprueba el regreso de la Bundesliga para finales de mayo". La Vanguardia, 42.

Rodríguez, Roberto. 2020b, 1 abril. "La FIA anuncia cambios para salvar a la fórmula 1 frente al coronavirus". La Vanguardia, 42.

Rojas-Torrijos, José Luis. 2012. "La futbolización de la información deportiva. Un estudio de casos de cuatro diarios deportivos europeos". Comunicação E Cultura, n. ${ }^{\circ}$ 13: 77-95. https://doi.org/ 10.34632/comunicacaoecultura.2012.629

Rojas-Torrijos, José Luis. 2018. "La estrategia digital de internacionalización de Marca en Latinoamérica. Estudio de caso de MARCA Claro en México". Revista de Comunicación 17, n. ${ }^{\circ}$ 1: 133-54. https://doi.org/10.26441/RC17.1-2018-A7

Rojas-Torrijos, José Luis. 2020, 14 marzo. "Un periodismo deportivo sin competiciones, una oportunidad para reinventarse", Periodismo deportivo de calidad, https://bit.ly/33PIJ55 (consultado el 12 julio 2020).

Rojas-Torrijos, José Luis y Miguel Ángel Jimeno. 2019. "La presencia de las mujeres deportistas en la prensa regional en España. Un análisis de la fotografía de portada”. Communication Papers 8, n. ${ }^{\circ}$ 16: 33-48. http://dx.doi.org/10.33115/udg_bib/cp.v8i16.22357

Rowe, David. 2007. "Sports journalism: Still the 'toy department' of the news media". Fournalism 8, n 4 : 385-405. https://doi.org/10.1177/1464884907078657 
Rowe, David. 2017. "Sports journalism and the FIFA scandal: Personalization, co-optation, and investigation". Communication $\mathcal{E}$ Sport 5, n. ${ }^{\circ}$ 5: 515-33. https://doi.org/ $10.1177 / 2167479516642206$

Ruipérez, Carles. 2020, 24 abril. "Rousaud da marcha atrás". La Vanguardia, 40.

Ruiz Olabuénaga, José Ignacio, Iratxe Aristegui y Leire Melgosa. 1998. Cómo elaborar un proyecto de investigación social. Bilbao: Universidad de Deusto

Sainz-de-Baranda, Clara. 2014. "Las mujeres en la prensa deportiva: dos perfiles". Cuadernos de Psicología del Deporte 14, n. ${ }^{\circ}$ 1: 91-102.

Sainz-de-Baranda, Clara, Miguel Ángel Barbero-González y José-Gabriel Fernández-Fernández. 2019. "Tratamiento informativo del bádminton y el piragüismo en Marca y El País (2012-2016)". Estudios sobre el Mensaje Periodístico 25, n. . 3: 1605-16. https://doi.org/10.5209/ esmp.67007

Salaverría, Ramón, Nataly Buslón, Fernando López-Pan, Bienvenido León, Ignacio López-Goñi y María-Carmen Erviti. "Desinformación en tiempos de pandemia: tipología de los bulos sobre la Covid-19". El profesional de la información 29, n. ${ }^{\circ}$ 3: 1-15. https://doi.org/10.3145/ epi.2020.may. 15

Schultz-Jørgensen, Søren. 2015. “The world's best advertising agency: the sports press". Mandag Morgen, n. ${ }^{\circ}$ 37: 1-7. https://bit.ly/2PXJS2F

Seoane, María Cruz y Susana Sueiro. 2004. Una historia de El País y del Grupo Prisa. Barcelona: Plaza \& Janés.

Serazio, Michael. 2019. The power of sports. Media and spectacle in American culture. Nueva York: New York University Press.

Solves, Josep, Athanasios Pappous, Inmaculada Rius y Geoffrey Zain Kohe. 2019. "Framing the Paralympic Games: A Mixed-Methods Analysis of Spanish Media Coverage of the Beijing 2008 and London 2012 Paralympic Games”. Communication E Sport 7, nº 6: 729-51. https:// doi.org/10.1177/2167479518808237

Steiner, Miriam, Melanie Magin y Birgit Stark. 2019. "Uneasy bedfellows. Comparing the diversity of German public service news on television and on facebook". Digital Fournalism 7, n. ${ }^{\circ}$ 1: 100-23. https://doi.org/10.1080/21670811.2017.1412800

Toro, Carlos. 2020, 22 marzo. "Lorenzo Sanz, el presidente de la Séptima”. El Mundo, 86.

Torres, Diego. 2020a, 1 mayo. "Francia declara campeón al PSG”. El País, 35.

Torres, Diego. 2020b, 25 abril. "Holanda declara desierto el título de la Eredivisie". El País, 31.

Torres, Diego. 2020c, 5 abril. "El experimento alemán”. El País, 43.

Tovar, Jorge. 2021. "Soccer, World War II and coronavirus: a comparative analysis of how the sport shut down". Soccer and Society 22, n. ${ }^{\circ}$ 1-2: 66-74. https://doi.org/ $10.1080 / 14660970.2020 .1755270$

Waisbord, Silvio. 2019. "The vulnerabilities of journalism". Fournalism 20, n. ${ }^{\circ}$ 1: 210-13. https:// doi.org/10.1177/1464884918809283 